\title{
Constrained Kalman Filter based Interference Suppression in Phased Arrays
}

\author{
P.D. Omgond and Hema Singh \\ Centre for Electromagnetics (CEM) \\ CSIR-National Aerospace Laboratories \\ Bangalore 560017 \\ Email: hemasingh@nal.res.in
}

\begin{abstract}
The constrained Kalman filter-based adaptive algorithm has fast convergence and robustness towards direction-of-arrival (DOA) mismatch. This motivates its usage for interference suppression in phased arrays. The constraints related to high gain towards the desired source and minimum mean square error are imposed in the weight adaptation. The objective is to steer the mainbeam towards the desired direction and simultaneously nullify the interfering sources impinging the array from different directions. It is shown that the output SINR of the algorithm is higher than the well-known gradient-based improved LMS algorithm.

Index Term - Adaptive array processing, conventional Kalman filter, constrained Kalman filter, mean square error, output SINR.
\end{abstract}

\section{INTRODUCTION}

The adaptive array processing involves interpretation of the impinging signals, weight adaptation to generate the desired array pattern. The role of adaptive algorithm, in principle, is to facilitate the generation of optimum antenna weights for a given signal scenario. The adapted pattern shows maximum gain towards the desired direction with nulls towards each of the interfering directions. In open domain various adaptive algorithms have been proposed and implemented. These algorithms make use of different constraints or criteria to adapt the phased array system towards high signal-tointerference and noise ratio (SINR) and steered beam towards signal of interest. An algorithm with less complexity, low computation cost, high convergence rate, low output noise power, and robust towards mismatch errors is in general preferred [1].

The optimal weight vector can be determined for a given desired signal vector by minimizing mean square error (MSE) using Kalman filter theory. The unconstrained Kalman filter had been employed in array processing [2]. This method however is sensitive to DOA mismatch errors and for the desired signals closely spaced with the hostile interfering signals. This motivates the development of constrained Kalman filter-based adaptive algorithm [3]. The array processing included non-linear constraints for weight adaptation in narrowband signal scenario. This approach enhanced the robustness against the pointing errors, imperfect array calibration, wavefront distortion, etc.

In this paper, the constrained Kalman filter based adaptive algorithm is employed to generate adapted pattern. The received signal vector due to impinging desired and interfering signals is used to generate array correlation matrix (ACM). The point constraint and constraint related to minimum MSE are incorporated in the array processing. The output noise power and SINR are used as performance indices to evaluate the array performance. The adapted pattern is generated for narrowband signal scenario. The efficiency of the constrained Kalman filter-based adaptive algorithm is compared with that of improved LMS algorithm.

\section{CONSTRAINED KALMAN FILTER BASED ADAPTIVE ALGORITHM}

Assume a signal scenario with one desired signal and $P$ probing sources. These signals along with additive zero mean Gaussian thermal noises are received by a uniform linear array (ULA) of $N$ antenna elements. The output of phased array system can be expressed as

$$
y(k)=x^{H}(k) \hat{w}(k) .
$$

where $x(k)=\left[x_{1}(k), \ldots, x_{N}(k)\right]^{T}$ is the received signal vector, consisting of desired signal, $s(k)$, interfering signals $i_{l}(k)$ for $l=1,2, \ldots N-1$ and thermal noise $n(k)$. The complex weight vector is $\hat{w}(k)=\left[\hat{w}_{1}(k), \ldots, \hat{w}_{N}(k)\right]^{T}$. Thus, the total received signal vector can be written as

$$
x(k)=S_{o} . s(k)+S_{l}(k) \cdot i_{l}(k)+n(k) .
$$

where $S_{o}$ is the steering vector for desired signal, $S_{l}$ is the steering vector for interfering signals. The impinging signals and the thermal noise are assumed to be uncorrelated.

The adapted pattern generated using conventional Kalman filter suffers from mainlobe distortions. In order to maintain the mainlobe towards the desired direction minimize the output noise power and hence maximize output SINR, the constraints are included in weight adaptation. The constraints are mathematically expressed as,

$$
\min _{W} w^{H} \widetilde{R}_{x x}(k) w \quad \text { subjected to } \quad w^{H} \cdot S=1 .
$$

where $S$ is steering vector towards desired source, and $\widetilde{R}_{x x}(k)$ is the array correlation matrix. The solution to (3) is given by [4], 


$$
\begin{array}{r}
f_{2}(w(k))=\varepsilon^{2} w^{H}(k) w(k)-w^{H}(k) S S^{H} w(k) \\
+w^{H}(k) S+S^{H} w(k)
\end{array}
$$

where $\varepsilon$ is Kalman robustness parameter. The constraint problem can thus be written as

$$
\min _{W} w^{H} \widetilde{R}_{x x}(k) w \quad \text { subjected to } f_{2}(w(k))=1 .
$$

A state vector $w$ of a array system can be updated as

$$
w(k)=\gamma w(k-1)+v_{p n}(k-1)
$$

where $\gamma$, a process parameter, plays an important role in the obtaining optimum weights, $v_{p n}$ is the process noise vector with covariance matrix $Q_{p}=\sigma_{p}^{2} I ; \sigma_{p}^{2}$ is the noise variance.

Incorporating the constraints into the weight adaptation, the measurement equation is expressed as

$$
\left[\begin{array}{l}
0 \\
1
\end{array}\right]=\left[\begin{array}{c}
x^{H}(k) w(k) \\
f_{2}(w(k))
\end{array}\right]+\left[\begin{array}{l}
u_{1}(k) \\
u_{2}(k)
\end{array}\right] .
$$

In matrix notation,

$$
z(k)=f(w(k))+u_{m}(k) .
$$

where $u_{m}$ is measurement noise vector, consisting of residual error, $u_{1}$ and the constraint error $u_{2}, z$ is $\mathrm{M} \times 1$ vector, $M$ being number of constraints considered. The measurement noise vector can be modeled as zero-mean, independent white noise sequences with the covariance matrix, given by

$$
Q_{m}=\left[\begin{array}{cc}
\sigma_{1}^{2} & 0 \\
0 & \sigma_{2}^{2}
\end{array}\right] \text {. }
$$

The weight vector is updated as [5]

$$
\hat{w}(k)=\hat{w}(k-1)+G(k)[z(k)-\hat{z}(k-1)]
$$

where the predicted measurement vector is given by

$$
\hat{z}(k-1)=E[f(w(k))]=\left[\begin{array}{c}
\gamma x^{H}(k) \hat{w}(k-1) \\
f_{2}(\gamma \hat{w}(k-1))+\frac{1}{2} \operatorname{Tr}\left\{H^{(2)} P(k-1)\right\}
\end{array}\right]
$$

where $H$ is $\mathrm{M} \times \mathrm{N}$ Hessian matrix,

$$
H=\left[\begin{array}{l}
H^{(1)} \\
H^{(2)}
\end{array}\right]=\left[\begin{array}{c}
0 \\
\varepsilon^{2} I-S S^{H}
\end{array}\right] .
$$

The predicted weight vector covariance matrix, $P(k-1)$ is given by

$$
P(k-1)=\gamma^{2} P(k-1 \mid k-1)+Q_{p} .
$$

The iteration start with initial weight vector $w(0)=S_{0} / N$, and covariance matrix due to weight vector $\mathrm{P}(0 \mid 0)=\beta I$.

The Kalman gain matrix $G(k)$ in (10) is obtained as

$$
G(k)=P(k-1) J_{w}^{H}(k, \gamma \hat{w}(k-1)) S_{i n}(k)^{-1} .
$$

where, $J_{w}(k, w(k))$ is the Jacobian of $f(w(k))$, expressed as

$$
J_{w}(k, w(k))=\frac{\left[\partial f^{H}(w(k))\right]^{H}}{\partial w(k)}=\left[\begin{array}{c}
x^{H}(k) \\
\varepsilon^{2} w^{H}(k)-\left(S S^{H} w(k)\right)^{H}+S^{H}
\end{array}\right]
$$

where $S_{i n}(k)$ is innovation covariance matrix is expressed as

$$
\begin{aligned}
S_{i n}(k) & =J_{w}(k, \gamma \hat{w}(k-1)) P(k-1) J_{w}^{H}(k, \gamma \hat{w}(k-1)) \\
& +\frac{1}{4}\left[\begin{array}{ll}
0 & 0 \\
0 & 1
\end{array}\right] \operatorname{Tr}\left\{H^{(2)} P(k-1) H^{(2)} P(k-1)\right\}+Q_{m} .
\end{aligned}
$$

The covariance matrix due to updated weight vector is given by

$$
\begin{aligned}
P(k)= & \left\{I-G(k) J_{w}(k, \gamma \hat{w}(k-1))\right\} P(k-1)\left\{I-G(k) J_{w}(k, \gamma \hat{w}(k-1))\right\}^{H} \\
& +\frac{1}{4} G(k) T r\left[H \cdot P(k-1) H^{H} P^{H}(k-1)\right] G^{H}(k)+G(k) Q_{m} G^{H}(k) .
\end{aligned}
$$

This updated covariance matrix will be used for next iteration until optimum weights are obtained towards converged output noise power and SINR.

\section{RESULTS AND DISCUSSION}

A uniform linear array of ten antenna elements spaced halfa-wavelength apart is considered to receive uncorrelated, narrow-band signals. The received thermal noise is modeled as zero mean, Gaussian noise with covariance of 0.04 . The Kalman robustness parameter $\varepsilon$ is taken as 3 . The residual and constrained errors are modeled as zero mean, uncorrelated white noise sequences with variance $10^{-10}$ and $10^{-12}$ respectively. Fig. 1 shows the comparison between the output SINR of constrained Kalman filter based adaptive algorithm and improved LMS algorithm for one desired $\left(0^{\circ}, 1 \mathrm{~dB}\right)$ and one interfering signal $\left(50^{\circ}, 30 \mathrm{~dB}\right)$. It may be observed that in case of constrained Kalman filter based algorithm, output SINR converges to higher value ( $22 \mathrm{~dB})$ within 100 snapshots.

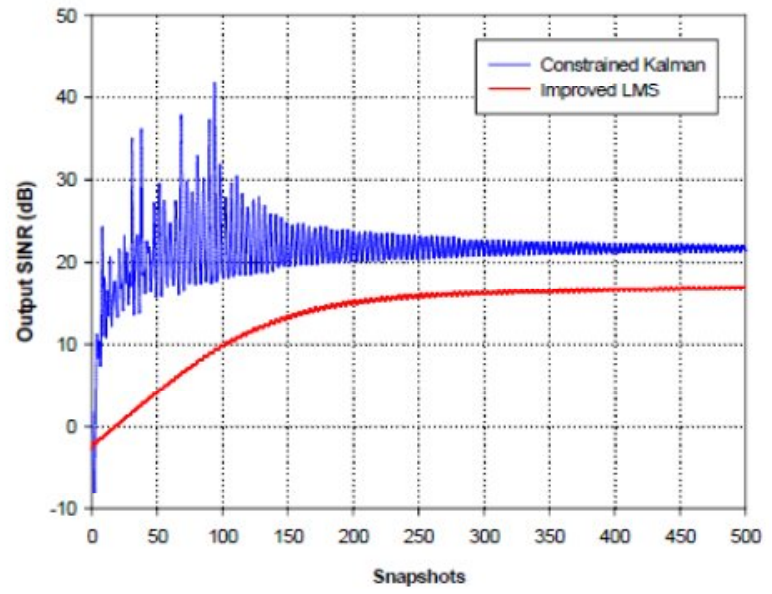

Fig. 1. Output SINR of ten-element linear array. One desired signal $\left(0^{\circ} ; 1 \mathrm{~dB}\right)$ and one interfering source $\left(50^{\circ} ; 30 \mathrm{~dB}\right)$.

However in the case of improved LMS algorithm output SINR converges to $16 \mathrm{~dB}$ in 250 snapshots. The constrained 
Kalman algorithm shows better performance both in terms of convergence rate and converged output SINR value. Fig. 2 shows corresponding adapted beam pattern generated using two algorithms. It is apparent that both the algorithms maintain mainlobe towards the desired source with accurate deep null towards the interfering source. However the null depth in case of constrained Kalman filter based algorithm is deeper $(-69 \mathrm{~dB})$ as compared to that of improved LMS algorithm $(-57 \mathrm{~dB})$. This shows the efficiency of the constrained Kalman filter based algorithm. It not only converges fast to high output SINR but also suppresses the interfering signal efficiently with mainlobe towards the desired source. Fig. 3 presents the variation of output SINR with SNR of desired source. The signal scenario considered consists of one desired signal $\left(0^{\circ}\right)$, two interfering sources $\left(30^{\circ}, 50^{\circ} ; 30 \mathrm{~dB}\right.$ each). It may be noted that except for few initial snapshots, the output SINR increases with increase in SNR of desired source.

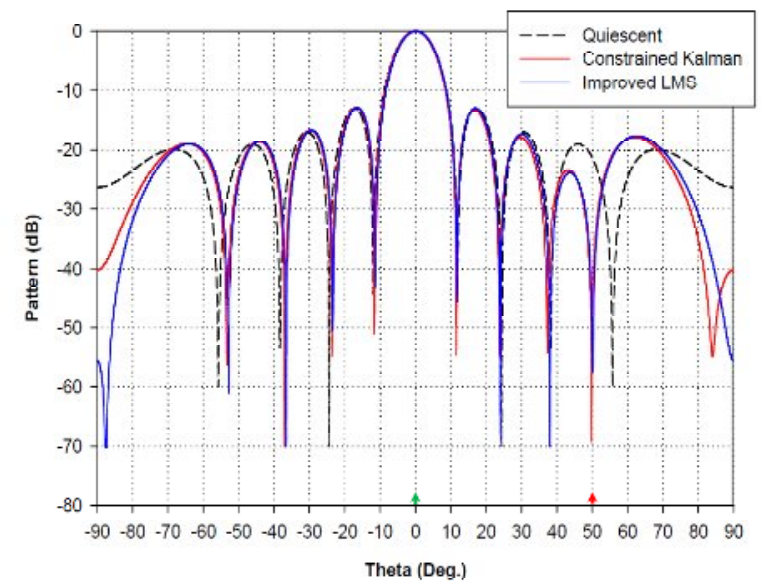

Fig. 2. Adapted beam pattern of ten-element linear array. One desired signal $\left(0^{\circ} ; 1 \mathrm{~dB}\right)$ and one interfering source $\left(50^{\circ} ; 30\right.$ $\mathrm{dB})$. The direction of the desired source is indicated with green arrow while interfering source is represented by red arrow.

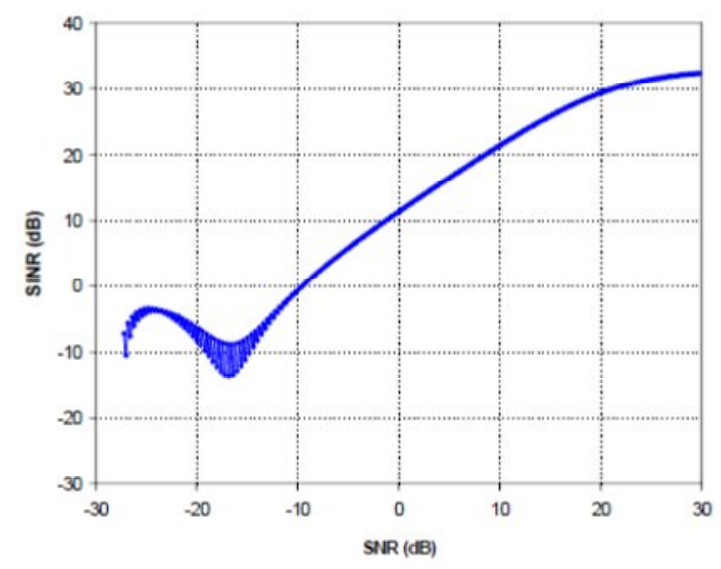

Fig. 3. Output SINR of ten-element linear array versus SNR of desired signal. The signal scenario: one desired signal $\left(0^{\circ}\right)$, two interfering sources $\left(30^{\circ}, 50^{\circ} ; 30 \mathrm{~dB}\right.$ each $) ; \gamma=0.1$.
Next a signal scenario consisting of one desired $\left(0^{\circ} ; 1 \mathrm{~dB}\right)$ and two interfering sources $\left(30^{\circ}, 45^{\circ} ; 30 \mathrm{~dB}\right.$ each) are considered to impinge the array. Fig. 4 shows the adapted and the quiescent patterns of the array. It may be observed that the array efficiently maintains the mainlobe towards the desired source with sufficiently deep nulls towards both the interfering signals.

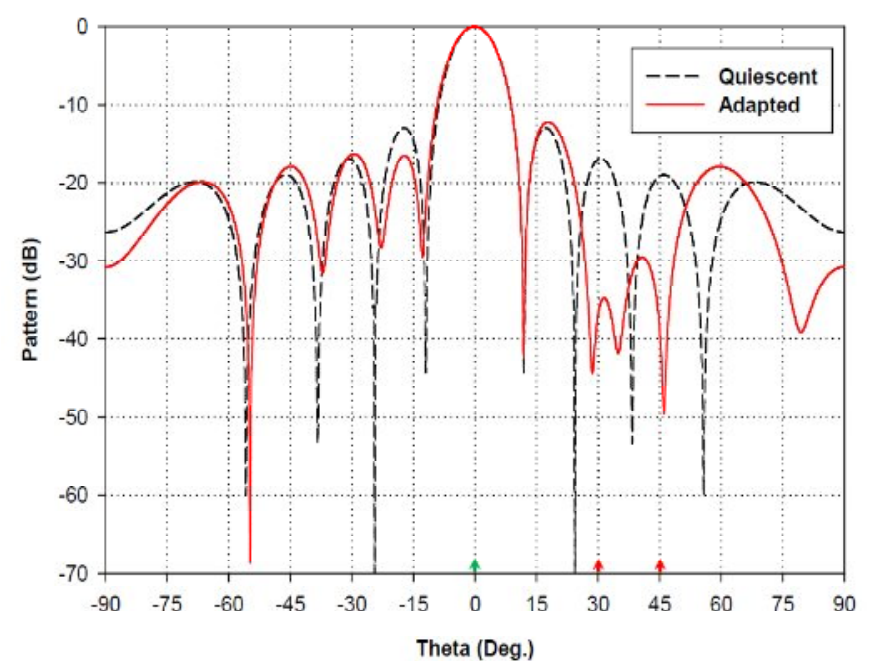

Fig. 4. Adapted beam pattern of ten elements linear array. One desired signal $\left(0^{\circ} ; 1 \mathrm{~dB}\right)$ and two interfering sources $\left(30^{\circ}, 45^{\circ} ; 30\right.$ $\mathrm{dB}$ each); $\gamma=0.4$.

\section{CONCLUSION}

The weight adaptation for uniform linear array is carried out using constrained Kalman filter based adaptive algorithm. The optimal weights obtained are used to compute the adapted pattern and output SINR. The results demonstrate the efficiency of the constrained Kalman filter algorithm in catering arbitrary signal environment. The adapted pattern generated maintains mainlobe towards the desired source and consists of sufficiently deep nulls in the interfering signal directions.

\section{REFERENCES}

[1] M. S. Razzaq, and N. M. Khan, "Performance comparison of adaptive beamforming algorithms for smart antenna systems," World Applied Sc. Journal, vol. 11, no. 7, pp. 775-785, 2010.

[2] Y. H. Chen, and C. T. Chiang, "Adaptive beamforming using the constrained Kalman filter," IEEE Transactions on Antennas and Propagation, vol. 41, no. 11, pp. 1576-1580, Nov. 1993.

[3] A. E. Keyi, T. Kirubarajan, and A. B. Gershman, "Robust adaptive beamforming based on the Kalman filter," IEEE Trans. Signal Process., vol. 53, no. 8, pp. 3032-3041, Aug. 2005.

[4] S. A. Vorobyov, A. B. Gershman, and Z. Q. Luo, "Robust adaptive beamforming using worst-case performance optimization: A solution to the signal mismatch problem," IEEE Trans. Signal Process., vol. 51, no. 2, pp. 313-324, Feb. 2003.

[5] M. H. Hayes, Statistical Digital Signal Processing and Modeling, New York: John Wiley \& Sons, 608 p., 1996. 